\title{
Antibody and antibody mimetic immunotherapeutics
}

\author{
"Rarely has a therapeutic approach been the focus of so much \\ attention, generated so much enthusiasm (inside and outside of \\ the laboratory), and developed so rapidly - both within the global \\ pharmaceutical industry and academic laboratories.
}

First draft submitted: 7 March 2017; Accepted for publication: 14 March 2017; Published online: 3 August 2017

Keywords: activation immunotherapeutic $\bullet$ antibody $\bullet$ biologics $\bullet$ immunotherapy

- nanobody • suppression immunotherapeutic

\section{What are immunotherapeutics?}

Immunotherapy is an approach to treating disease that relies on controlling the immune system. Immunotherapeutics are classified as either suppression immunotherapeutics, which reduce the immune response, or activation immunotherapeutics, which elicit the immune system to seek out and destroy diseased cells. Immunotherapy emerged as a field and therapeutic strategy with the development and administration of immune system suppressing small-molecule drugs (e.g., azathioprine, FK506, cyclosporin A and rapamycin). As immune system suppressants, these drugs have been used to treat inflammatory and autoimmune diseases, as well as to suppress rejection following organ transplant. Today, immunotherapeutics continue to be used to treat a multitude of inflammatory and autoimmune diseases. Over the last two decades, however, immunotherapeutics have also been developed to elicit immune system components to seek out and destroy numerous cancers.

Rarely has a therapeutic approach been the focus of so much attention, generated so much enthusiasm (inside and outside of the laboratory), and developed so rapidly - both within the global pharmaceutical industry and academic laboratories. It is difficult to overstate the impact immunotherapeutics have on human health, and their place in modern pharmaceuticals. Of the top ten selling drugs in 2015 [1], six are characterized, at least in part, as having an immunotherapeutic mechanism of action (Humira ${ }^{\mathrm{TM}}$, Enbrel ${ }^{\mathrm{TM}}$, Remicade $^{\mathrm{TM}}$, Rituxan ${ }^{\mathrm{TM}}$, Herceptin ${ }^{\mathrm{TM}}$ and RevlimidTM). $^{\mathrm{TM}}$.

\section{Biologics as immunotherapeutics}

In addition to witnessing an explosion of immunotherapeutics into the market, the past two decades have also witnessed the age of biologics - principally protein drugs. Historically, disease has been treated with small-molecule drugs. However, in contrast to their small-molecule counterparts, the size and complexity of proteins often result in surfaces capable of recognizing diseaserelevant receptors that challenge or evade small molecules. Additionally, proteins can be evolved (either in vivo or in the laboratory) to selectively and potently bind virtually any disease-relevant receptor. This initial discovery process is often much simpler, and less expensive, than the analogous small-molecule centered approach. By virtue of these facts, it is perhaps unsurprising that advances

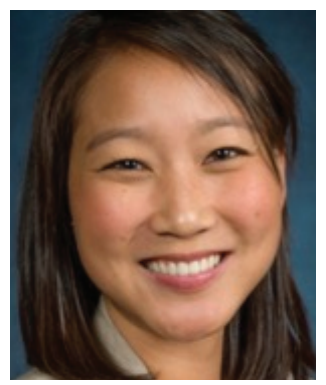

Angeline $\mathrm{N} \mathrm{Ta}$

Department of Chemistry, Colorado State University, Fort Collins, CO 80523 , USA

and

Department of Biochemistry \& Molecular Biology, Colorado State University, Fort Collins, CO 80523, USA

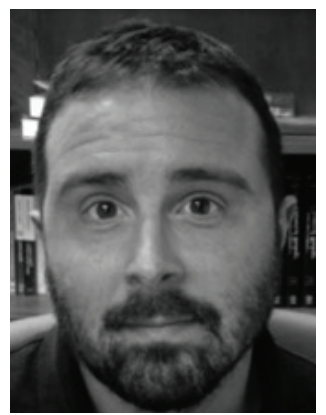

Brian R McNaughton Author for correspondence: Department of Chemistry, Colorado State University, Fort Collins, CO 80523, USA

and

Department of Biochemistry \& Molecular Biology, Colorado State University, Fort Collins, CO 80523, USA mcnaughton@colostate.edu

newlands Press part of 
in immunotherapy and biologics have dovetailed - and this relationship has led to new immunotherapeutics and drug leads.

\section{Antibody immunotherapeutics}

Principally, biological immunotherapeutics are fulllength immunoglobulins of the isotype $\mathrm{G}$ ( IgG, referred to herein as antibodies). These include topselling drugs Humira, Remicade, Rituxan and Herceptin. Antibodies are large $(\sim 150 \mathrm{kDa})$ multidomain proteins [2]. In the context of immunotherapeutic activity and pharmacology, antibodies have two important regions. Recognition of a binding partner occurs within the fragment antigen-binding (Fab) region, which consists of heavy- and light-chain domains. Specifically, binding interactions are the result of sequence optimization of loops, so-called complimentary determining regions (CDRs) within the variable light-chain and variable heavy-chain domains $\left(\mathrm{V}_{\mathrm{L}}\right.$ and $\mathrm{V}_{\mathrm{H}}$, respectively) [2]. Some antibodies can act as suppression immunotherapeutics by binding to, and thus sequestering, immune-stimulating polypeptides. For example, Humira is a full-length monoclonal antibody that binds to TNF- $\alpha$, which in the absence of Humira forms a complex with TNF- $\alpha$ receptors [3] and activates an inflammatory response. Given the ability of this drug to inhibit the TNF- $\alpha /$ TNF- $\alpha$ receptor complex, Humira is an effective treatment for autoimmune and inflammatory diseases, including rheumatoid arthritis, psoriatic arthritis, ankylosing spondylitis, Crohn's disease, plaque psoriasis, ulcerative colitis, chronic psoriasis, hidradenitis suppurativa, juvenile idiopathic arthritis and noninfection uveitis. Similarly, Remicade is a TNF- $\alpha$ binding antibody, and thus an effective treatment for many autoimmune and inflammatory diseases [4].

\footnotetext{
"While regions within full-length antibodies enable tailored recognition, long serum stability and activation immunotherapeutic activity, the complex structure and post-translational modifications of these molecular behemoths " complicate their production and manipulation."
}

The in vivo half-life of antibodies (ca. 21 days) is much longer than most proteins [2]. This virtue is endowed by an epitope in the fragment crystallizable region $(\mathrm{Fc})$, which binds the neonatal $\mathrm{Fc}$ receptor, FcRn, in a pH-dependent manner. Formation of the Fc/FcRn complex leads to a complicated process that continuously shuttles the antibody from the circulatory system to the cell interior (within endosomes) and back again. As a result of this biological shell game, antibodies evade degradation by serum and lysosomal proteases $[5,6]$. A region found within $\mathrm{Fc}_{\mathrm{c}}$ is also necessary to induce activation immunotherapeutic mechanisms, such as antibody-dependent cellular cytotoxicity (ADCC) $[7,8]$ or complement-dependent cytotoxicity (CDC) [9,10]. Specifically, Fc $\gamma$-receptors on the surface of immune effector cells, such as natural killer cells and macrophages, recognize $\mathrm{Fc}_{\mathrm{c}}$ in antibodies that are bound to disease-relevant receptors on the surface of targeted cells (via interactions involving CDRs within the Fab fragment). Following Fc-Fc $\gamma$-receptor complex formation, the immunoreceptor tyrosine-based activation motif is phosphorylated, triggering activation of the effector cell and release of perforin, lytic enzymes, TNF and/or granzymes for cell destruction through ADCC. In CDC, C1q of complex $\mathrm{C} 1$ binds to the $\mathrm{Fc}$ region and triggers the complement cascade activation and eventual formation of membrane attack complex at the surface of the target cells. Ultimately, this leads to lysis of the antibody-bound cell.

Current activation immunotherapeutics include Herceptin, a monoclonal antibody that binds to an extracellular domain of HER2, a biomarker that is overexpressed on the surface of approximately $20-30 \%$ of breast cancers and gastric cancers [11]. In addition to other therapeutic mechanisms, experiments on laboratory animals show that Herceptin recruits immune system components to HER2-positive cells, resulting in cell-selective ADCC. In a similar vein, Rituxan is a monoclonal antibody that binds CD20 on the surface of normal and malignant B cells, leading to, among other outcomes, ADCC and CDC [12]. As an activation immunotherapeutic that facilitates selective destruction of B cells, Rituxan principally finds use in fighting cancers of the blood, such as non-Hodgkin's lymphoma and chronic lymphocytic leukemia. However, the story is more complex: Rituxan can also act as a suppression immunotherapeutic. Since Rituxan leads to destruction of $\mathrm{B}$ cells - a critical component of the immune system - this drug suppresses the immune response. As a result, Rituxan is also an effective therapeutic for a number of autoimmune diseases such as rheumatoid arthritis.

\section{Antibody mimetic immunotherapeutics}

While regions within full-length antibodies enable tailored recognition, long serum stability and activation immunotherapeutic activity, the complex structure and post-translational modifications of these molecular behemoths complicate their production and manipulation. In response, researchers have developed minimalist antibodies and antibody mimetics. A number of small $(<20 \mathrm{kDa})$ proteins that mimic CDR display within antibody Fab fragments have been reported. Evolution of these CDR loop mimics, 
using methods like phage display, often leads to new proteins with tailored recognition - including recognition of disease-relevant cell surface biomarkers. Popular examples include monobodies [13] - a fibronectin type III-derived protein scaffold - and affimers [14] - a phytocystatin-derived protein scaffold. In addition to these nonantibody scaffolds, researchers have relied on nanobodies [15] - the fragment antigen-binding region in camelid-derived heavy-chain $\operatorname{IgG}$ (which lacks a light chain). In contrast to antibodies, these proteins often have a relatively small number of disulfide bonds (or none at all), are not post-translationally glycosylated, express well in bacteria or yeast and are relatively stable. Collectively, these features simplify their production and manipulation. In addition, their small size, relative to full-length antibodies, can result in improved tumor penetration [16].

Antibody mimetics are often simpler to prepare and manipulate, but lack Fc. As a result, these proteins experience relatively short lifetimes in vivo, and do not induce or activate the immune system. Recently, however, protein engineers have used techniques such as sequence-selective chemical conjugation and/or genetic fusion as a means to generate nonantibody proteins with dramatically improved in vivo stability, and potent activation immunotherapeutic activity.

One approach to turning on activation immunotherapeutic activity is to incorporate a component (such as a small molecule) that recruits an endogenous antibody (which contains Fc and thus can recruit immune effector cells). Fortunately, a number of small molecules have been reported that bind endogenous antibodies. For example, researchers have shown that antibodies recognizing dinitrophenyl (DNP) constitute approximately $1 \%$ of circulating IgM and approximately $0.8 \%$ of circulating IgG, likely due to exposure to DNP-containing dyes, preservatives and/or pesticides. Recently, we showed that sequence-selective bioconjugation of a DNP containing molecule to a HER2-binding nanobody led to a new immunotherapeutic that potently and selectively destroys HER2-positive breast cancer cells in culture, via ADCC [17].

Of course, since nanobodies lack Fc, these therapeutic leads undoubtedly have relatively short in vivo stability compared with IgG antibodies. However, a number of strategies have been reported that dramatically improve the in vivo stability of nonantibody proteins. These include chemical conjugation to PEG (PEGylation), fusion to $\mathrm{Fc}$ or genetic fusion to human serum albumin (HSA)-binding peptides or proteins [18]. The development of nanobody-based immunotherapeutics with improved serum stability is a major focus of many pharmaceutical companies. For example, recently Ablynx reported nanobody-based immunosuppressant drug leads, which are now in clinical trials. Since nanobodies are amenable to extensive mutagenesis and manipulation, scientists have been able to genetically link them to proteins that endow properties, such as long in vivo stability, and multivalent recognition. For example, Vobarilizumab $^{\mathrm{TM}}$ consists of an IL-6R-binding nanobody that is genetically fused to an HSA-binding nanobody [19]. Recognition of IL-6R blocks the IL-6R/IL-6 interaction, leading to a blockage of the inflammatory response. In concert, the HSA-binding nanobody component allows the drug to find residence on HSA, an abundant $(35-50 \mathrm{~g} / \mathrm{l})$ protein in blood. As a result, the HSA-bound drug essentially hides from serum proteases and enjoys a relatively long lifetime in vivo. Similarly, Ablynx has also reported a nanobody-based immunotherapeutic that targets two different diseaserelevant receptors. ALX-0761 (M1095) is an anti-IL$17 \mathrm{~A} / \mathrm{F}$ nanobody designed to treat autoimmune disorders such as psoriasis [20]. This nanobody-based fusion protein consists of an anti-IL-17F nanobody, anti-HSA nanobody and anti-IL-17A/F nanobody - resulting in a $40-\mathrm{kDa}$ trivalent suppression immunotherapeutic that neutralizes proinflammatory cytokines, IL-17A and IL-17F. While optimization of nanobody-based fusions, such as overcoming sequestration of smaller proteins (MW $<40 \mathrm{kDa}$ ) by the kidneys, is certainly necessary, this new frontier in immunotherapeutic design and discovery will undoubtedly lead to new therapies.

\section{Conclusion}

Decades ago, a handful of small-molecule drugs capable of suppressing the immune system ignited the field of immunotherapy. As molecular and cellular biology techniques rapidly evolved, enabling cloning and large-scale production of proteins, recombinant antibody drugs joined the effort. As a result, immunotherapeutics represent a significant - and growing - sector of the global pharmaceutical industry. Most recently, researchers with expertise in protein engineering and bioconjugation are developing new antibody mimetics with long serum stability and/or activation immunotherapeutic activity. These biologics represent a new class of suppression or activation immunotherapeutics, and will undoubtedly have a significant impact on the future of human health.

\section{Financial \& competing interests disclosure}

The authors have no relevant affiliations or financial involvement with any organization or entity with a financial interest in or financial conflict with the subject matter or materials discussed in the manuscript. This includes employment, consultancies, honoraria, stock ownership or options, expert testimony, grants or patents received or pending, or royalties.

No writing assistance was utilized in the production of this manuscript. 


\section{References}

1 Pharma Compass. Top drugs by sales revenue in 2015: who sold the biggest blockbuster drugs? (2016).

www.pharmacompass.com

2 Bruce VJ, Ta AN, McNaughton BR. Minimalist antibodies and mimetics: an update and recent applications. Chembiochem 17(20), 1892-1899 (2016).

3 Menter A, Tyring SK, Gordon K et al. Adalimumab therapy for moderate to severe psoriasis: a randomized, controlled Phase III trial. J. Am. Acad. Dermatol. 58(1), 106-115 (2008).

4 Keane J, Gershon S, Wise RP et al. Tuberculosis associated with infliximab, a tumor necrosis factor (alpha)-neutralizing agent. N. Engl. J. Med. 345(15), 1098-1104 (2001).

5 Goebl NA, Babbey CM, Datta-Mannan A, Witcher DR, Wroblewski VJ, Dunn KW. Neonatal Fc receptor mediates internalization of $\mathrm{Fc}$ in transfected human endothelial cells. Mol. Biol. Cell 19(12), 5490-5505 (2008).

6 Roopenian DC, Akilesh S. FcRn: the neonatal Fc receptor comes of age. Nat. Rev. Immunol. 7(9), 715-725 (2007).

7 Ravetch JV, Clynes RA. Divergent roles for Fc receptors and complement in vivo. Annu. Rev. Immunol. 16, 421-432 (1998).

8 Seidel UJ, Schlegel P, Lang P. Natural killer cell mediated antibody-dependent cellular cytotoxicity in tumor immunotherapy with therapeutic antibodies. Front. Immunol. 4(76), 1-8 (2013).

9 Prang N, Preithner S, Brischwein K et al. Cellular and complement-dependent cytotoxicity of Ep-CAM-specific monoclonal antibody MT201 against breast cancer cell lines. Br. J. Cancer 92 (2), 342-349 (2005).

10 Walport MJ. Advances in immunology: complement (first of two parts). N. Engl. J. Med. 344(14), 1058-1066 (2001).

11 Hudis CA. Drug therapy: trastuzumab - mechanism of action and use in clinical practice. N. Engl. J. Med. 357(1), 39-51 (2007).
12 Edwards JCW, Szczepanski L, Szechinski J et al. Efficacy of B-cell-targeted therapy with rituximab in patients with rheumatoid arthritis. N. Engl. J. Med. 350 (25), 2572-2581 (2004).

13 Koide A, Bailey CW, Huang XL, Koide S. The fibronectin type III domain as a scaffold for novel binding proteins. J. Mol. Biol. 284(4), 1141-1151 (1998).

14 Woodman R, Yeh JTH, Laurenson S, Ferrigno PK. Design and validation of a neutral protein scaffold for the presentation of peptide aptamers. J. Mol. Biol. 352(5), 1118-1133 (2005).

15 Muyldermans S. Nanobodies: natural single-domain antibodies. Annu. Rev. Biochem. 82, 775-797 (2013).

16 Holliger P, Hudson PJ. Engineered antibody fragments and the rise of single domains. Nat. Biotechnol. 23(9), 1126-1136 (2005).

17 Gray MA, Tao RN, Deporter SM, Spiegel DA, Mcnaughton BR. A nanobody activation immunotherapeutic that selectively destroys HER2-positive breast cancer cells. Chembiochem 17(2), 155-158 (2016).

18 Vazquez-Lombardi R, Phan TG, Zimmermann C, Lowe D, Jermutus L, Christ D. Challenges and opportunities for non-antibody scaffold drugs. Drug Discov. Today 20(10), 1271-1283 (2015).

19 Van Roy M, Ververken C, Beirnaert E et al. The preclinical pharmacology of the high affinity anti-IL-6R nanobody (R) ALX-0061 supports its clinical development in rheumatoid arthritis. Arthritis Res. Ther. 17, 135 (2015).

20 Vanheusden K, Detalle L, Hemeryck A et al. Pre-clinical proof-of-concept of ALX-0761, a Nanobody ${ }^{\circledR}$ neutralizing both IL-17A and $\mathrm{F}$ in a cynomolgus monkey collagen induced arthritis model. Presented at: 2013 ACR/ARHP Annual Meeting. San Diego, CA, USA, 25-30 October 2013 (Abstract 1287). 